\title{
Free Space Optical Communications: An Overview
}

\author{
M. N. O. Sadiku \\ S.M. Musa \\ College of Engineering, \\ Prairie View A\&M University, Prairie View, TX \\ Sudarshan R. Nelatury \\ School of Engineering and Engineering Technology \\ Pennsylvania State University Erie, PA
}

\begin{abstract}
Bridging the so-called "last mile" in communication networks has revived keen interest in free-Space Optics (FSO), also known as fiber-free or fiberless optics, which is a technology that transports data via laser technology. It is a line-of-sight technology that currently enables optical transmission up to $2.5 \mathrm{Gbps}$ of data, voice and video through the air at long distances $(4 \mathrm{~km})$, allowing optical connectivity without deploying fiber-optic cable or securing spectrum licenses. It is moving closer to being a realistic alternative to laying fiber in access networks. This paper presents an introduction to FSO and the current state of its technology.
\end{abstract}

Keywords: Communication networks, optical communication

\section{Introduction}

Spectrum scarcity, coupled with bandwidth appetites in metropolitan area networks (MANs), is forcing wireless operators and service provides to look at new methods to connect cells. They are faced with many options in attempts to meet the high bandwidth demand. The first and often times most obvious choice is fiber-optic cable, but the associated delays and costs to lay fiber often make it economically prohibitive. The second alternative is radio frequency (RF) technology, which is a mature technology for longer distance transmission than FSO, but RF technologies cannot scale to optical capacities of $2.5 \mathrm{Gbps}$. The current RF bandwidth ceiling is $622 \mathrm{Mbps}$. The third option is copper-based technologies such as T1, cable modem, or DSL. Although copper infrastructure is available almost everywhere, it is still not a viable alternative because the bandwidth limitation of 2 to $3 \mathrm{Mbps}$ makes it a marginal solution. The fourth and most viable choice is FSO. 
The technology is an optimal solution, given its optical base, bandwidth scalability, speed of deployment, portability, and cost-effectiveness (Willebrand and Ghuman, 2002; Chan, 2006; Wang et al., 2015; Yang et al., 2014).

Free-space optics (FSO), also known as fiber-free or fiberless photonics, refers to the transmission of modulated light pulses through free space (air or the atmosphere) to obtain broadband communications. Laser beams are generally used, although non-lasing sources such as light-emitting diodes (LEDs) or IR-emitting diodes (IREDs) will serve the same purpose. FSO can be the best wireless solution where fiber optical cable is not available, high bandwidth (anywhere from $1 \mathrm{Mbps}$ up to $1.25 \mathrm{Gbps}$ ) is required, and line-of-sight can be obtained to a target within a couple of miles.

Since FSO is a convergence of two disparate technologies, it is not clear whether it is a wireless or optical system. FSO is an optical technology and not a wireless technology for two basic reasons. First, FSO enables optical transmission at speeds of up to $2.5 \mathrm{Gbps}$ and in the future $10 \mathrm{Gbps}$ using WDM. This is not possible using any fixed wireless/RF technology existing today. Second, FSO technology requires no FCC licensing or municipal license approvals and thus obviates the need to buy expensive spectrum. This distinguishes it clearly from fixed wireless technologies.

This paper takes a closer look at FSO technology, its strengths and drawbacks. It examines how FSO is responding to high bandwidth communication needs in the metro area and how FSO beats competing local access alternatives such as DSL.

\section{Free space optics}

FSO is not new. It was developed more than three decades ago. Then, it was used by the military and space aviation pioneers to provide secure and rapidly deployable communications links. For example, it is being used to carry data within digital computing systems (Gourlay et al., 1998), in cross-bar switching (Rajkumar et al., 1996), optical interconnections (Jahns, 1994), and optoelectronic sampling (Wu and Zhang, 1997). Recent developments in optical technology have advanced FSO to mainstream communications applications and make it an alternative to RF wireless.

\section{Conventional Optics vs. Free Space Optics}

Optical fiber transmission has been the dominant technology for the past decade for data communications over long and medium distances (see Fig. 1). However, for short distances, optical communications has some technological disadvantages. As a result of this, optical communications has 
not been able to penetrate into areas such as chip-to-chip and board-to-board communication (Jahns, 1994).

Fiber-optic cable and FSO share some similarities. The theory of FSO is essentially the same as that for fiber optic transmission. The use of lasers is a simple concept similar to optical transmissions using fiber-optic cables. The only difference is the medium; the signal is sent through air or free space from the source to the destination, rather than guided through an optical fiber. Light travels through air faster than it does through glass. So one may regard FSO as optical communications at the speed of light.

Without a doubt, optical fiber is the most reliable means of providing optical communications. However, the digging, delays and associated costs to lay fiber often make it very expensive. Moreover, once fiber is deployed, it becomes a "sunk" cost and cannot be re-deployed if a customer needs to relocate or switch to another service provider, making it extremely difficult to recover the investment in a reasonable timeframe. Furthermore, most of the recent trenching to lay fiber has been to improve the metro core (backbone), while the metro access and edge have completely been ignored. Free space optical technologies offer an effective and economical way to address the "last mile" bottleneck by connecting to fiber backbone infrastructure directly to customer premises. FSO network is designed with short optical links (typically range from 200 to $2000 \mathrm{~m}$ ), whereas fiber optic cable can be used for long-haul ( up to $200 \mathrm{~km}$ without repeaters). It provides levels of bandwidth comparable to fiber optic cable. It promises high connectivity and dispersion-free dynamic optical paths - a feature that is lacking in fiber optic communication networks. FSO is compared with other access technologies in Table 1.

Table 1 Comparision of FSO with other access technologies (Buckley, 2001).

\begin{tabular}{|l|l|l|l|}
\hline Features & FSO & Fiber & DSL \\
\hline Deployment time & Days to weeks & $4-12$ months & $6-12$ months \\
\hline Provisioning time & Immediate & Complex & Complex \\
\hline $\begin{array}{l}\text { Initial investment for few } \\
\text { subscribers }\end{array}$ & Low & High & High \\
\hline Reliability & Medium & High & High \\
\hline Topology/flexibility & PP, PM, Mesh & PP, PM, Mesh & PP \\
\hline Distance Limitation & $200-2000 \mathrm{~m}$ & $200 \mathrm{~km}$ & $5.5 \mathrm{~km}$ \\
\hline Bandwidth/speed & $1.25 \mathrm{Gbps}$ & $10 \mathrm{Gbps}$ & $2 \mathrm{Mbps}$ \\
\hline
\end{tabular}

\section{FSO Technology}

Transmission using FSO technology is relatively simple. It involves two systems each comprising of an optical transceiver which consists of a laser transmitter and a receiver to provide full duplex (bi-directional) capability. FSO uses low-power lasers and a telescope to transmit single or multiple wavelengths through the air to a receiver . As illustrated in Fig. 2, 
each FSO system uses a high-power optical source such as laser or LED and a telescope that transmits light through the atmosphere to another telescope that receives the information. At that point, the receiving telescope connects to a high-sensitivity receiver through an optical fiber. At the source, the visible or IR energy is modulated with the data to be transmitted. At the destination, the beam is intercepted by a photodetector, the data is extracted from the visible or IR beam (demodulated), and the resulting signal is amplified and sent to the hardware. If the energy source does not produce a sufficiently parallel beam to travel the required distance, collimation can be done with lenses.

One can determine the coverage area of a typical FSO system using Fig. 3. The distance $d$, the incident light radius $r$, and the beam divergence angle $\theta$ are related as

$$
r=d * \tan \left(\frac{\theta}{2}\right)
$$

For example, for a beam divergence angle of $0.006 \mathrm{rad}, 2 \mathrm{r}=3.6 \mathrm{~m}$. FSO systems can function over distances of several hundred meters up to 4 $\mathrm{km}$, depending on system characteristics and environmental conditions. As long as there is a clear line-of-sight (LOS) between the source and the destination, communication is theoretically possible. Even if there is no direct line-of-sight, strategically positioned mirrors can be used to reflect the energy. The beams can pass through glass windows with little or no attenuation.

FSO technology has proven itself in other applications, particularly those requiring a tactical, point-to-point link. FSO's greatest success so far has come from the LAN/campus connectivity market. Such applications could include a link between a newsroom and a broadcasting station, or a dedicated link between two high-traffic nodes in a large building complex. Thus, to maintain quality of service for a particular customer, the distance from that customer's location to the nearest hub may have to be shortened.

\section{Key Strengths and Weaknesses}

FSO has many benefits besides its massive bandwidth. These include the following:

- It operates in a completely unregulated frequency spectrum ( range of $\mathrm{THz}$ ). Because there is little or no traffic currently in this range, the FCC has not required licenses above $600 \mathrm{GHz}$. This means FSO is not likely to interfere with other transmissions.

- Cost is a major advantage. Significantly lower cost on average than the construction of a new fiber optical solution, or leased lines. With FSO, there is also no capital overhang. 
- It can be deployed in days to weeks versus months to years (no excavation of sidewalks, building permits, etc.)

- $\quad$ Bandwidth can easily be scaled with virtually unlimited headroom (10 Mbps to $1.25 \mathrm{Gbps)}$ per link.

- $\quad$ An FSO network architecture need not be changed when other nodes (buildings) are added; customer capacity can be easily increased by changing the node numbers and configurations.

- $\quad$ The technology is easily upgradeable, and its open interfaces support equipment from a variety of vendors, which helps carriers protect the investment in their embedded infrastructures.

One would think that security is problematic with wireless, but FSO is fairly difficult to intercept. Because its beams are invisible, narrow, and very directional (aimed at a particular antenna), it is hard to find a particular traffic link in the air, let alone crack the code. In addition, links from customer to hub are typically encrypted.

Like any new technology, FSO does have its potential drawbacks, which will be discussed later.

In spite of these drawbacks, however, FSO is poised to become a major player in the local broadband access market, particularly among small and medium-size businesses, which typically lack fiber connections.

\section{Free-space optical networks}

Free space optical networking technologies provide an effective and economically compelling solution to the "last mile" problem of connecting to fiber infrastructure in metropolitan areas. Free-space optical networking technology enables businesses to transmit and receive data transmission among buildings up to 2.5 miles apart at speeds much faster than a typical high-speed/leased line.

\section{Architecture Options}

Like other networks, FSO networks can assume different configurations depending on the end user's needs and the desired application. As shown in Fig. 4, there are four common topologies are point-to-point, point-to-multipoint, a mesh, and a ring. In a point-to-point arrangement, FSO can support speeds between $155 \mathrm{Mbps}$ to $10 \mathrm{Gbps}$ at a distance of 2 to $4 \mathrm{~km}$. A point-to-multipoint or star configuration involves multiple links originating from a single node and it can support the same speeds at a distance of 1 to $2 \mathrm{~km}$. A mesh topology can support $622 \mathrm{Mbps}$ at a distance of $200 \mathrm{~m}$ to $450 \mathrm{~m}$. It is possible to combine these topologies. Generally, point-to-point link provides a dedicated connection with higher bandwidth, but does not scale cost-effectively. Point-to-multipoint is cheaper but offers less bandwidth. Point-to-multipoint systems suffer not only from the single 
point-of-failure problem but also from costs related to hub development, lower reliability (because of longer distances), and issues with hub location, which is critical for maximizing the number of buildings within LOS. Mesh architectures are most useful, because they can transmit data to a node from several directions, avoiding an obstructed path if necessary. Their chief benefit is service restoration (redundancy) via multiple network nodes. The tradeoff is that the distance covered declines. Because of the need for scalability, most analysts would prefer a mesh topology, which allows carriers to add nodes to the network more easily. The mesh also allows for alternate routing, while other topologies suffer from a single point of failure. The ring is the common topology used by the metropolitan service providers. The backbone is represented by high-speed rings, which are fiber or FSO based. A typical application of point-to-point link connecting two fast Ethernet-based networks is shown in Fig. 5. A typical installation is on 4.1 $\mathrm{km}$ path which is essentially horizontal and lies $50 \mathrm{~m}$ above the street level with the wavelength of the carrier $\lambda=830 \mathrm{~nm}$ (Jahns, 1994).

\section{Major Market Drivers}

The market forces behind FSO networks include (Willebrand and Ghuman, 2002; Nykolak, 1999; LightPointe; Epple and Henniger, 2007):

(1) Increasing demand for bandwidth: Demand for bandwidth has been increasing exponentially for the past few years. Service providers have been struggling to keep up with such high demand, with DWDM being used to meet that need.

(2) Increasing Internet traffic: The Internet is generating a great need for high bandwidth at the edge of the network. The number of Internet users is increasing daily and is expected to grow to about 796 millions by 2005 .

(3) Increasing e-commerce: With the growing number of businesses involved in e-commerce activities, e-commerce is fast becoming a user of high bandwidth.

(4) High capacity desktops: With multimedia and an exponential increase in processor speeds, the desktop computer is now an enabler of high bandwidth applications.

(5) Upgrading of MAN: Deploying DWDM-based optical metropolitan area networks (MANs) and upgrading them is a direct result of the increase in bandwidth usage at the edge.

(6) Advances in optics: New developments in fiber optical devices are making broadband free-space optical transmission an appealing alternative to RF wireless, and a flexible cost-effective adjunct to optical fiber as well. Due to these recent advances, fiber optic technologies have made the transition from use in expensive long-haul communication medium to low- 
cost medium between computing systems and peripherals, local area networks (LANs), and other computers.

Free-space optics offers a cost-effective, quick and available infrastructure that is not only easily deployed (within days), redeployed, and easy to manage, but can also offer a multitude of options-distance, speed, topology and installations.

\section{Deployment}

The Strategis Group foresees an exponential deployment of FSO, as evident in its forecast of FSO equipment revenue for 1999 to 2005 shown in Table 2. FSO equipment currently is being deployed for a variety of applications, such as (Willebrand and Ghuman, 2001):

1. Last-mile access: FSO can be deployed in high-speed links that connect end-users with Internet service providers or other networks.

2. Metro network extensions: FSO can be deployed by carriers to extend their MAN fiber rings.

3. Enterprise connectivity: FSO can be used to interconnect LAN segments in buildings.

4. Fiber backup: FSO can be used as redundant link in place of a second fiber link.

Among these applications, last-mile access may provide the greatest opportunity since FSO provides the high-speed links that customers need without the costs of laying fiber.

Table 2 FSO Equipment Revenue.

\begin{tabular}{lccccccc} 
Year & 1999 & 2000 & 2001 & 2002 & 2003 & 2004 & 2005 \\
\hline Revenue (\$millions) & 1.7 & 51.4 & 111.7 & 199.8 & 354.1 & 579.2 & 864.9 \\
\hline
\end{tabular}

Source: The Strategis Group

The early adopters of FSO include the Smithsonian Institution, Barclays Bank, and the New York City Fire Department. The next generation of adopters will include carriers that can move swiftly and decisively, utilizing FSO as a valuable tool for connecting customers who demand bandwidth that cannot be met any other way.

To speed up the deployment of FSO, the Free Space Optical Alliance (www.fsoalliance.com) was been formed in February, 2001 and had it first meeting in Boston in June, 2001. It primary Mission is to provide a unified FSO "strategy position" to the outside technical community and promote FSO as a viable broadband technology for metro-access networks, backhaul private networks, and ultimately even residential applications. The FSO Alliance is comprised of 13 leading companies that provide FSO systems 
and service providers. Its member include AirFiber, AT\&T, Canon, fSONA, LightPointe, Optical Access, Plaintree, Qwest, Teligent, Texas Instrument, and Zyoptics.

Despite its potential, FSO still has many challenges to overcome before it will be deployed widely. These challenges include (Buckley, 2001; Willebrand and Ghuman, 2001):

- Weather: FSO is an LOS technology, meaning that nodes must have an unobstructed path to the hub antenna. In other words, interference of any kind can pose problems. One form of interference is the weather condition. A major challenge to FSO communications is fog. As shown in Table 3, there could be to $300 \mathrm{~dB}$ per kilometer of attenuation on foggy days. Snow or rain does not bother FSO much, because the light can find its way through the raindrops and snowflakes, but fog can stop lasers like a brick wall. Fog is vapor composed of water droplets, which are only a few hundred microns in diameter but can modify light characteristics or completely hinder the passage of light through a combination of absorption, scattering and reflection.

Table 3 Typical weather attenuation (Willebrand and Ghuman, 2001).

\begin{tabular}{cc}
\hline \hline Weather Conditions & Attenuation \\
\hline Clear & 5 to $15 \mathrm{~dB} / \mathrm{km}$ \\
Rain & 20 to $50 \mathrm{~dB} / \mathrm{km}$ \\
Snow & 50 to $150 \mathrm{~dB} / \mathrm{km}$ \\
Fog & 50 to $300 \mathrm{~dB} / \mathrm{km}$ \\
\hline
\end{tabular}

Source: AirFiber

- Absorption: Absorption occurs when suspended water molecules in the terrestrial atmosphere extinguish photons. This causes a decrease in the power density (attenuation) of the FSO beam and directly affects the availability of a system. Absorption occurs more readily at some wavelengths than others.

- Scattering: Scattering is caused when the wavelength collides with the scatterer like flying birds, building, structures, and towers. The physical size of the scatterer determines the type of scattering. Unlike absorption, there is no loss of energy in scattering, only a directional redistribution of energy that may have significant reduction in beam intensity for longer distances.

- $\quad$ Scintillation: Heated air rising from the earth or man-made devices such as heating ducts creates temperature variations with different air pockets. This can cause fluctuations in signal amplitude which leads to "image dancing" at the FSO receiver end. One way of minimizing the effect 
of scintillation is to have no links longer than $500 \mathrm{~m}$ and mount systems farther away from vents, hot roofs, and air-conditioning ducts.

- Safety: The two major concerns typically expressed involve questions about human exposure to laser beams (which present much more danger to the eyes than any other part of the human body) and high voltages within the laser systems and their power supplies. Several standards have been developed covering the performance of laser equipment and the safe use of lasers.

Vendors, such as Airfiber, LightPointe, and Lucent are addressing these problems. However, the biggest obstacle facing FSO may be its reputation or lack of one. Many people understand RF technology due to its wide use in mobile phones but lasers still remain a mystery to many.

However, FSO technology is not for everyone. FSO deployments must be located relatively close to big hubs and are therefore confined to urban areas, which means only customers in major cities will be eligible, at least initially.

\section{Conclusion}

The growth of communications networks has accelerated last-mile access needs for high speed links. Free Space Optics is now a viable choice for connecting the LAN, WAN, and MAN; and carrying voice, video and data at the speed of light. However, FSO links in the mid-infrared spectrum seemed to be more favorable as lower atmospheric transmission losses increase the reliability of the system, particularly under bad weather conditions with low visibility (Martini et al., 2002).

While fiber-optic communication has gained acceptance in the telecommunications industry, FSO communication is still a relatively new entrant. Its apprehension has not been universal; its development activity has been concentrated in the US. Its primary advantages are high throughput, solid security, and low cost. With current availability of up to $1.25 \mathrm{Gbps}$, throughputs of hundreds of Gbps are possible in the future. Free space optics is a technology that is poised for exponential growth in the coming years.

\section{References:}

Buckley, S. (2001). Free Space Optics: The Missing Link, Telecommunications, (Americas ed.), 35(10), 26-33.

Chaimowicz, J.C.A. and Cole, R.S., (1989). "Electronically steerable free space optical communication beams," $2^{\text {nd }}$ IEE National Conference on Telecommunications, $98-105$.

Chan, V. W. S. (2006). Free-space optical communications, Journal of Lightwave Technology, 24 (12),. 4750-4762. 
Epple B. and Henniger, H., (2007). Discussion on design aspects for freespace optical communication terminals, IEEE Communications Magazine, 62-69.

Gourlay, J. et al., (1998). Development of Free-Space Digital Optics in Computing, IEEE Computer, 38-44.

Jahns, J. (1994). Planar packaging of free-space optical interconnections, Proceedings of IEEE, 82(11), 1623-1631.

LightPointe, White papers developed by LightPointe and available at http://www.lightpointe.com/index.cfm

Martini, R. et al., (2002). Free-space optical transmission of multimedia satellite data streams using mid-infrared quantum cascade lasers, Electronics Letters, 38(4) 181-183.

Nykolak, G. et al., (1999). 2.5 Gbit/s free space optical link over 4.4 km, Electronics Letters, 36(7), 578-579.

Rajkumar, N. et al., (1996). Optoelectronic cross-bar switching using freespace optical distribution, IEEE Photonics Technology Letters, 8(11), 15341536.

Wang, Y., Wang, D. and Ma, J. (2015). On the performance of coherent OFDM systems in free-space optical communications, IEEE Photonics Journal, 7(4).

Willebrand, H. and Ghuman, B., (2001). Fiber optics without fiber, IEEE Spectrum, 41-45.

Willebrand, H. and Ghuman, B. (2002). Free Space Optics: Enabling Optical Connectivity in Today's Networks. Indianapolis, IN: SAMS.

Wu, Q. and Zhang, X.C., (1997). Free-space electro-optics sampling of midinfrared pulses, Applied Physics Letters, 71(10), 1285-1286.

Yang, L., X. Gao, and M. S. Alouini, (2014). Performance analysis of freespace optical communication systems with multiuser diversity over atmospheric turbulence channels, IEEE Photonics Journal, 6(2). 


\section{Distance}

\begin{tabular}{|c|c|c|}
\hline \multirow{2}{*}{$10^{6} \mathrm{~m}$} & Technology & Application \\
\hline & Fiber optics & $\begin{array}{l}\text { Wide area } \\
\text { networks }\end{array}$ \\
\hline $10^{3} \mathrm{~m}$ & Fib & \\
\hline $10^{0} \mathrm{~m}$ & $\begin{array}{l}\text { Waveguide } \\
\text { optics } \\
\text { FSO }\end{array}$ & $\begin{array}{l}\text { Metropolitan } \\
\text { area } \\
\text { networks }\end{array}$ \\
\hline $10^{-3} \mathrm{~m}$ & & Local area \\
\hline
\end{tabular}

Fig. 1 Hierarchy of optical communication technologies and applications (Gourlay et al., 1998). 


\section{Free space}

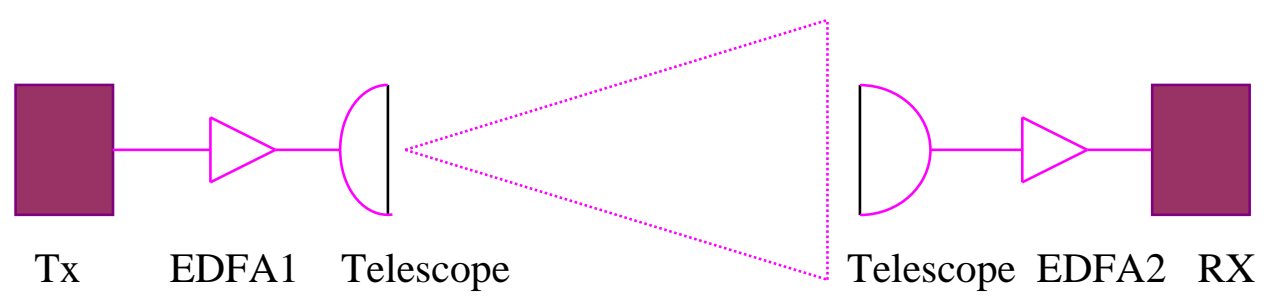

Fig. 2 Schematic diagram of a typical free-space optical system.

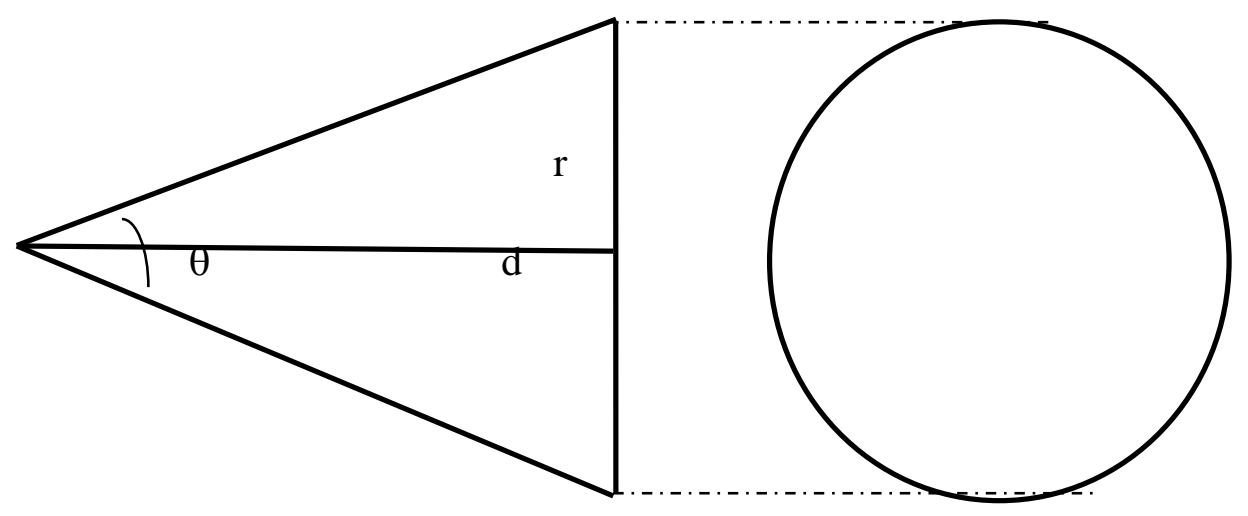

Fig. 3 Relationship between distance and beam divergence. 

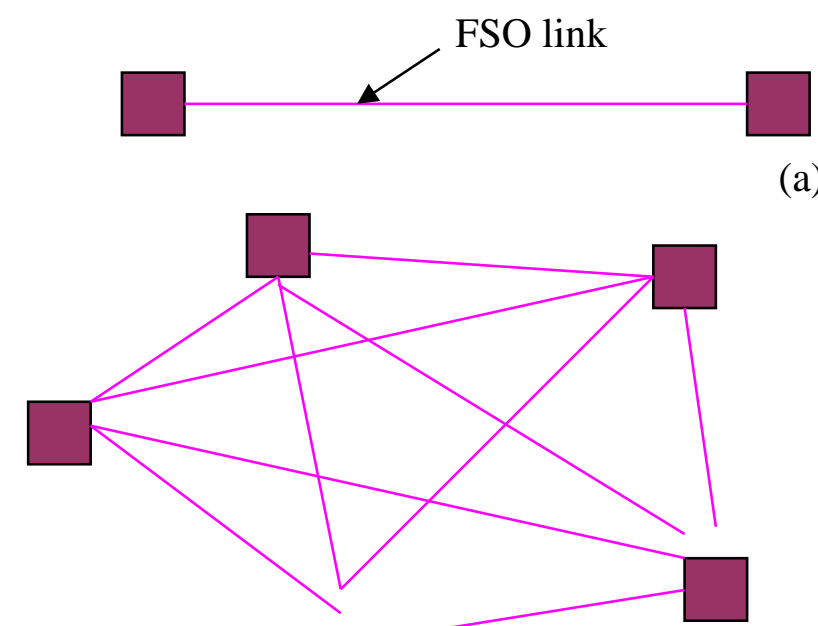

(a)

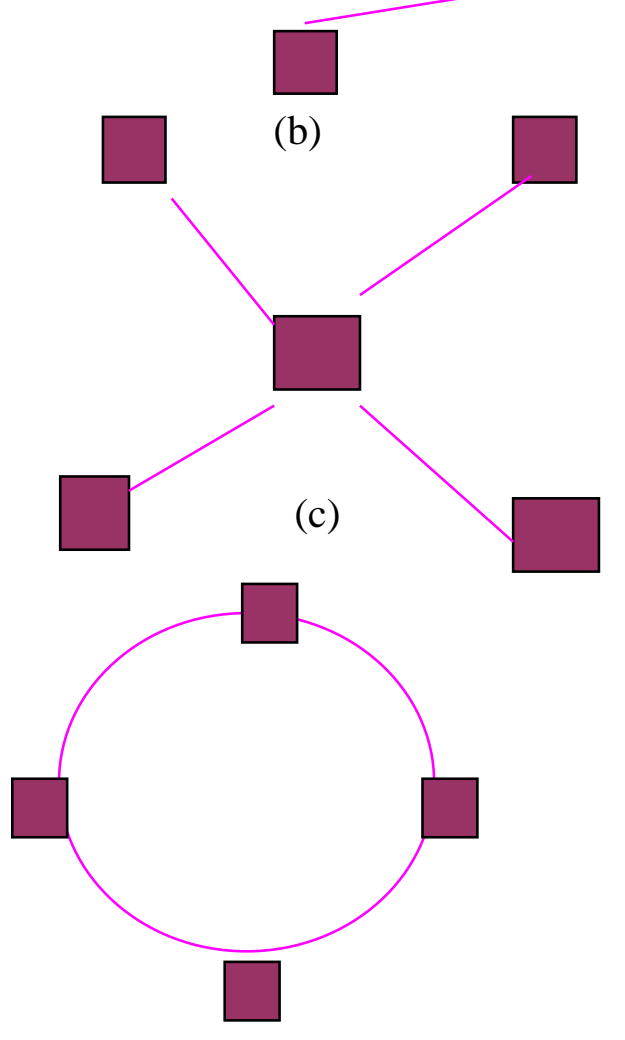

(d)

Fig. 4 FSO architectures: (a) point-point, (b) mesh, (c) point-multipoint or star, (d) ring. 


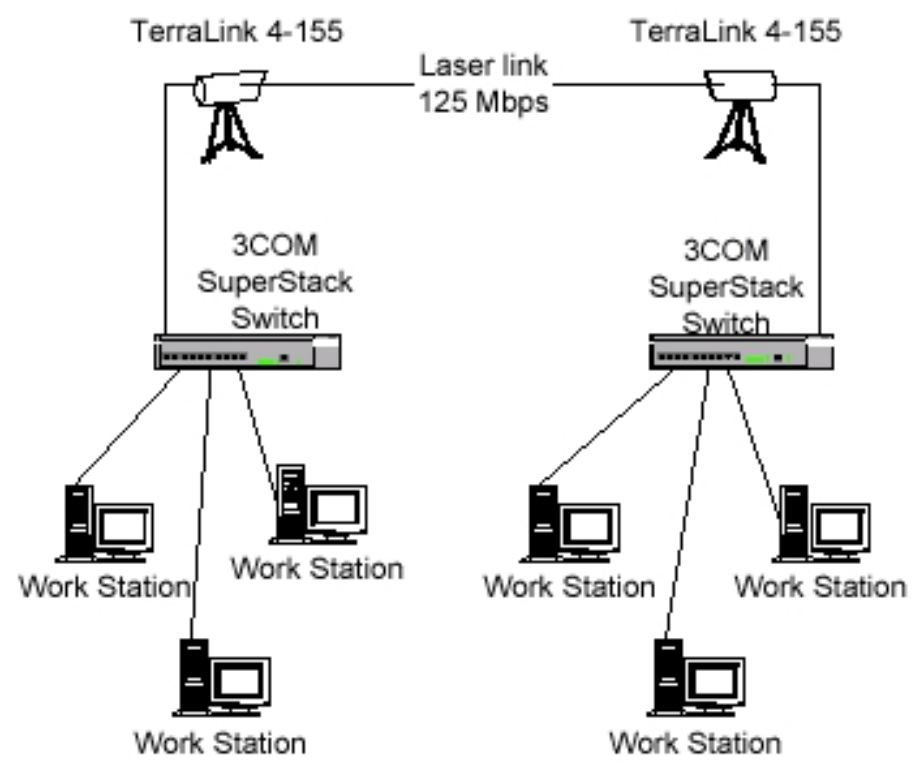

Fig. 5. Fast Ethernet Example (Source: Optical Access) 